improvements in death notification can be achieved in EMRO countries using a clear notification form and simple quality assurance process.

\section{P1-172 CANCER EPIDEMIOLOGY IN THE NORTH OF TUNISIA 1999-2003}

doi:10.1136/jech.2011.142976d.65

${ }^{1}$ W H B Ayoub, ${ }^{2} \mathrm{H}$ Rais, ${ }^{*}{ }^{1} \mathrm{~S}$ Zehani, ${ }^{3} \mathrm{H}$ Hsairi, ${ }^{3} \mathrm{~N}$ Achour, ${ }^{1} \mathrm{M}$ B Abdallah. ${ }^{1}$ Department of epidemiology and bio statistics, Salah Azaiez Institute of oncology, Tunis, Tunisia; ${ }^{2}$ Medical oncology department, Salah Azaiez Institute of oncology, Tunis, Tunisia; ${ }^{3}$ National Institute of Public Health, Tunis, Tunisia

Introduction Tunisia as a developing country is passing through an epidemiologic changing with less infectious diseases and the occurrence of several other chronic diseases.Cancer is becoming a major problem of public health. The registry of cancer in Tunisia started in 1997 by the creation of the cancer registry of Northern Tunisia (CRNT), a population registry involving about half the population. This study aimed to present data of the CRNT including the epidemiologic profile and the evolution of the incidence rate of most cancers sites during a 10 year period (1994-2003) in the north of the country.

Methods Referring to the CRNT data,we were able to determine the repartition of cancer according to sex, to calculate the brut and standardised incidence rate with a direct method on the basis of a world reference population. We could also find the incidence evolution and the cancer number of cases between 1994 and 2003. The results were compared to other registries data.

Results From 1999 to 2003, the CRNT registered the average of 5049 cases per annum of invasive cancers. This average was about 3744 in 1994. The increase was about $36 \%$ in a 10 year period. The brut incidence rate was 120.2 in men and 94.8 in women. If standardised on age according to a referent world population, the result stayed the same (133.2 v/s101.4). Lung cancer occurred first in men and then bladder cancer. In women, the increased brut incidences concerned breast cancer and colon cancer, cervical cancer occupied the fourth position after the skin cancer. Over the period (1994-2003), the increase of $36 \%$ in cancer occurrence is due in men to the $60 \%$ increase of lung, colon and prostate cancer cases and in women the increase of $50 \%$ of breast cancer new cases and then $10 \%$ of colon cancer cases.

Conclusion It seems that there is a continuous increasing of some cancers in Tunisia: breast cancer in women and lung cancer in men, colon cancer for both.

\section{P1-173 DELAY IN THE FIRST DENTAL VISIT IN A BRAZILIAN COHORT STUDY}

doi:10.1136/jech.2011.142976d.66

M Saraiva, H Bettiol, M A Barbieri, L Holanda. * University of São Paulo, Ribeirão Preto, Brazil

Introduction In Brazil about $55 \%$ of 6 -yrs-old children, even in the highest socioeconomic levels, had never been to a dentist. The aim of this study was to investigate factors associated with the delay in the first dental visit (DDV).

Methods We used data from the follow-up (2004) of the birth cohort study of Ribeirão Preto (1994), including $1 / 3$ of the original sample $(n=790)$. DDV was defined as the age at the first dental visit after 6 yrs-old. Covariates used were maternal age, education and skin colour, marital status, number of siblings and household members and health insurance. Bivariate and stratified analysis was followed by a logistic regression with hierarchical modelling.
Results Overall, $55.5 \%$ had not been to a dentist by the age of 6 . The final model showed that DFV was negatively associated with private health insurance (OR 0.67 ; $95 \%$ CI 0.46 to 0.97 ) and with mother's education. Compared to mothers with university degrees the OR for those with <4 years of schooling was 11.0 (95\% CI 4.66 to 26.24 ) and the OR for those with at least 8 yrs of education was 5.49 (95\% CI 2.65 to 11.39 ). Moreover, DFV was positively associated with mothers age ( $>20$ compared to $£ 20$ yrs-old) 1.77 (95\% CI 1.1 to 2.83) and with the $\mathrm{n}$ umber of household members ( $>4$ compared with $<4$ members) 1.73 (95\% CI 1.09 to 2.75 ).

Conclusions Considering the universal system studies are necessary to understand the barriers mothers with low educational level to take their children to the dentist. Grant Fapesp 00/09508-7.

\section{P1-174 EPIDEMIOLOGY OF NON-FATAL MACHINE INJURY IN BANGLADESH}

doi:10.1136/jech.2011.142976d.67

${ }^{1} \mathrm{M}$ J Hossain, ${ }^{*} \mathrm{~A}$ K M F Rahman, ${ }^{1,3} \mathrm{~K}$ Ul Baset, ${ }^{1} \mathrm{~A}$ Biswas, ${ }^{1,2} \mathrm{~S}$ Shafinaz, ${ }^{1} \mathrm{~S}$ R Mashreky. ${ }^{1}$ Centre for Injury Prevention and Research, Bangladesh, Dhaka, Bangladesh; ${ }^{2}$ UNICEF, Dhaka, Bangladesh; ${ }^{3}$ University of West England, Bristol, UK

Objective To examine the incidence and characteristics of non-fatal machine injury in Bangladesh.

Methods A population-based cross-sectional survey was conducted between January and December 2003 in Bangladesh. Nationally representative data were collected from 171366 rural and urban households, with a total sample size of 819429 .

Results The incidence of non-fatal machine injury was 41.1 per 100000 per year. The rate was 6.95 times higher in Male than in females. Those who are age 15 to 19 years are most vulnerable groups than those of others. Rural people were at more than 2.15 times higher risk of machine injury than urban people. The average number of workdays lost was 262.57 (SD 419.741). The average duration of assistance required in daily living activities was 70.47 days (SD 227.324) days. The hospitalisation rate was 20.87 per 100000 population per year. The mean duration of hospital stay was 18.49 days. The rate of permanent disability was 6.71 per 100000 population-years.

Conclusion Machine injury is one of the major causes of morbidity, disability and workday loss Bangladeshi population. Adolescent workers are at highest risk. Factory and agricultural sector are the most common place of machine injury. Home is also third common place for machine related injury. To reduce this devastating health issue, a national strategy and Programme for machine injury prevention must be developed.

\section{P1-175 PREDICTING THE FUTURE BURDEN OF OCCUPATIONAL CANCER}

doi:10.1136/jech.2011.142976d.68

S Hutchings, ${ }^{*}$ L Rushton. Imperial College London, London, UK

Introduction Interventions to reduce occupationally related cancers should be evidence based. We have developed a method for forecasting the future burden of occupational cancer given past and projected exposure trends and under targeted reductions in workplace exposure levels.

Methods The method builds on an approach using attributable fractions (AFs) developed to estimate the current burden of occupational cancer. We project risk exposure periods (REPs), accounting for cancer latencies of up to 50 years, forward in time, to estimate $\mathrm{AFs}$ for a series of forecast target years given past and projected exposure trends and under targeted reduction scenarios. Adjustment 
factors for changes in exposed numbers and levels are applied in estimation intervals within the REPs.

Results The methods are illustrated using a range of scenarios for reducing lung cancer due to occupational exposure to respirable crystalline silica (RCS). AFs for lung cancer due to RCS could potentially be reduced from $2.07 \%$ in 2010 to nearly zero by 2060 , depending on the timing and success of interventions.

Conclusion The importance of focusing on achieving compliance with current exposure standards in small industries is highlighted and can be more effective than setting standards at a lower level. The method can be used to highlight high-risk carcinogens, industries and occupations and is designed to allow comparative estimates to be made even where exposure data are limited. It is adaptable for other countries and other exposure situations in the general environment and can be extended to include socio-economic impact assessment.

\section{P1-176 BIAS ESTIMATION METHODS FOR THE BURDEN OF OCCUPATIONAL CANCER}

doi:10.1136/jech.2011.142976d.69

S Hutchings, ${ }^{*}$ L Rushton. Imperial College London, London, UK

Introduction Bias occurs in particular when estimates are based on sparse or inadequate data. We have estimated the burden of cancer in Great Britain attributable to occupation using an attributable fraction (AF) methodology, and present an adaptation of Greenland's ${ }^{1}$ Monte-Carlo sensitivity analysis (MCSA) to account for bias uncertainty.

Methods Sources of bias in burden estimation include using Levin's estimator with adjusted RR, unknown cancer latency, unknown proportions exposed and inadequate estimates of employment turnover. Each source of bias operates on a component of the AF estimator, which is represented by a factor for which a prior distribution is estimated from independent sources. Monte-Carlo repeated sampling from these distributions is then used, recalculating the AF each time.

Results Results are presented graphically for a hierarchy of bias sources that contribute to an overall credibility interval for the AF. For sinonasal cancer and wood dust the intervals for bias due to the variables contributing to the proportions exposed are narrower than the interval for $\mathrm{RR}$ random error only, and bias from incorrect use of Levin's estimator makes the least contribution.

Conclusion The method presented illustrates the use of credibility intervals to indicate important sources of uncertainty and facilitates identification of data gaps and future research needs.

\section{REFERENCES}

1. Greenland S. Interval estimation by simulation as an alternative to and extension of confidence intervals. Int J Epidemiol 2004;33:1389-97.

2. Greenland S. Multiple-bias modelling for analysis of observational data. J Royal Statist Soc Series A 2005;168(Part 2):267-306.

\section{P1-177 DEVELOPMENT AND EVALUATION OF A FORECASTING MODEL FOR INFECTIOUS DISEASES IN JAPAN USING TIME-SERIES ANALYSIS}

doi:10.1136/jech.2011.142976d.70

${ }^{1} \mathrm{M}$ Inoue, ${ }^{*} \mathrm{~S}$ Hasegawa, ${ }^{3} \mathrm{~A}$ Suyama. ${ }^{1}$ Tottori University, Yonago, Japan; ${ }^{2}$ Hokkaido Institute of Public Health, Sapporo, Japan; ${ }^{3}$ Radiation Effects Research Foundation, Nagasaki, Japan

Having forecasts for infectious diseases can help support risk management and effective intervention against the outbreak of disease. The main object of this study was to obtain a suitable way of making predictions of disease incidents through the use of timeseries analysis.

Methods Retrospective data of Japanese infectious diseases were collected for the period of 2000-2010. In this study, influenza, mumps and infectious gastroenteritis were used for analysis. These data were separated into two groups: one (2000-2007) was designated the "training" set and the other (2008-2010), the "validation" set. We applied three models: an exponential smoothing method (ESM), an autoregressive integrated moving average (ARIMA) and a nearest neighbour method (NNM), to make predictions on the morbidity of the diseases. Statistical analysis for the ESM and the ARIMA model were carried out using SPSS Ver.19. The NNM was executed using a computer program made by us based on its' algorithm. We used the mean absolute percentage error (MAPE) to measure and quantify how well the data matched or "fit". For example, a lower MAPE value would indicate a better fit of the data. Results The best-fit model for influenza was the NNM, where the MAPE was $70 \%$. For mumps and infectious gastroenteritis, the ARIMA revealed the best fit, where the MAPE for these were $7 \%$ and $16 \%$, respectively.

Conclusion We found that the ARIMA and NNM provide a useful way of making predictions of disease. The models could well be used in planning for risk management against infectious diseases.

\section{P1-178 HETEROGENEITY OF THE 23-VALENT PNEUMOCOCCAL POLYSACCHARIDE VACCINE EFFICACY DUE TO VARIOUS UNDERLYING CONDITIONS OF CHRONIC PULMONARY DISEASE IN ELDERLY PATIENTS}

doi:10.1136/jech.2011.142976d.71

${ }^{1} \mathrm{~S}$ Inoue, ${ }^{2} \mathrm{~T}$ Kaneko, ${ }^{2} \mathrm{~S}$ Morita, ${ }^{3} \mathrm{Y}$ Natsumeda, ${ }^{1} \mathrm{~S}$ Mizushima. ${ }^{1}$ Yokohama City University, Graduate School of Medicine, Department of Epidemiology and Public Health, Yokohama, Japan, ${ }^{2}$ Yokohama City University, Graduate School of Medicine, Department of Biostatistics and Epidemiology, Yokohama, Japan; ${ }^{3}$ Yokohama City University, Graduate School of Medicine, Laboratory for Clinical Research, Yokohama, Japan

Introduction The efficacy of 23-valent pneumococcal polysaccharide vaccine (PPV23) against pneumonia in elderly with chronic illness remains controversial.

Methods A total of 1378 elderly outpatients with chronic pulmonary disease were informed of the PPV23 efficacy on infectious pulmonary exacerbations. Individual patients who responded affirmatively were vaccinated between August and November 2002 and rest of them was assigned for control. The prevaccine period was defined as August 2001-August 2002. The events of interest were the first episode of bacterial pulmonary infection and death from any cause. The participants were observed for 2 years after vaccination.

Results Frequent episodes of pulmonary infection in the prevaccine period increased the event rate significantly (no episode $17.3 \%$, one episode $48.3 \%$, more than one episode $74.6 \%$, $p<0.001$, the KaplanMeier method). No significant differences of the survival probability were observed between the vaccinated and unvaccinated group during the analysis of the whole cohort. The mortality rate was higher in the vaccinated group $(p<0.010)$. This was due to the higher vaccination rate in patients who had pulmonary infection during the prevaccine period $(p<0.001)$. No harmful effects of PPV23 on mortality rate was seen $(p=0.100)$ in the COX proportional hazards regression model. In subgroup analysis, PPV23 reduced event rate significantly only in patients with chronic respiratory failure who had not pulmonary infection during the prevaccine period $(p<0.019)$

Conclusion The PPV23 needs to be given to elderly patients with chronic pulmonary disease at an earlier time where infectious complication in the lung has not occurred yet. 